Board of Governors of the Federal Reserve System

International Finance Discussion Papers

Number 528

October 1995

\title{
ON THE INVERSE OF THE COVARIANCE MATRIX IN PORTFOLIO ANALYSIS
}

\author{
Guy V.G. Stevens
}

NOTE: In:ernational Finance Discussion Papers are preliminary materials circulated to stimulate discussion and critical comment. References in publications to International Finance Discussion Papers (other than an acknowledgment that the writer has had access to unpublished material) should be cleared with the author or authors. 


\begin{abstract}
The goal of this study is the derivation and application of a direct characterization of the inverse of the covariance matrix central to portfolio analysis. As argued below, such a specification of the inverse, in terms of a few primitive constructs, helps clarify the determinants of such key concepts as (1) the optimal holding of a given risky asset, (2) the slope of the risk-return efficiency locus faced by the individual investor, and (3) the pricing of risky assets in the Capital Asset Pricing Model. The two building blocks of the inverse turn out to be the non-diversifiable part of each asset's variance and the multiple regression and correlation coefficients obtained by regressing each asset's excess expected return on the set of excess expected returns of all other assets.
\end{abstract}




\title{
ON TCHE INVERSE OF THE COVARIANCE MATRIX IN PORTFOLIO ANALYSIS
}

\author{
Guy V. G. Stevens ${ }^{1}$
}

\section{Introduction and Summary}

The: goal of this study is the derivation and application of a direct characterization of the inverse of the covariance matrix $\left[\sigma_{i j}\right]$ central to portfolio analysis. As argued below, such a specification of the inverse, in terms of a few primitive constructs, helps clarify the determinants of such key concepts as (1) the optimal holding of a given risky asset, (2) the slope of the risk-return efficiency locus faced by the individual investor, and (3) the pricing of risky assets in the Capital Asset Pricing Model. The two building blocks of the inverse turn out to be the non-diversifiable part of each asset's variance, and the multiple regression and correlation coefficients obtained by regressing each asset's excess expected return on the set of excess expected returns of all other assets.

\section{Preliminaries}

It is well known that every optimizing mean-variance investor will choose a portfolio falling on his or her risk-return efficiency frontier -- the locus of portfolios of minimum variance conditional on a given expected return. As shown by Mossin (1973) and others, with the existence of a riskless asset, this frontier is a straight line in mean-standard deviation space, with a slope, $d E / d S$, equal to $\left(\bar{m}^{\prime} C^{-1} \bar{m}\right)^{1 / 2}$-- where $C^{-1}$, the inverse of the matrix of asset variances and covariances, is the subject of this paper, and $\bar{m}$ (and its transpose, $\bar{m}^{\prime}$ ) is the vector of the excess expected return of each asset over the riskfree rate: $\bar{r}_{i}-r_{f}$. To illustrate this derivation, assume markets for $N$ risky assets, each with stochastic return $\tilde{r}_{i}$ and expected return $\bar{r}_{i}$, along with the opportunity for unlimited lending and

1 The author is a Senior Economist in the Division of International Finance, Board of Governors of the Federal Reserve System. The views expressed in this paper are the author's and should not be interpreted as reflecting those of the Board of Governors or other members of its staff. My thanks go to Charles Thomas, John Ammer, and William Helkie for very helpful discussions and comments. 
borrowing of a riskless asset with return $r_{f}$. For an investor with initial wealth $W$, the expected return on any portfolio, $E(Y)$, can be defined as:

$$
E(Y)=\bar{Y}=r_{f} W+\sum_{i=1}^{N} z_{i}\left(\bar{r}_{i}-r_{f}\right)=r_{f} W+z^{\prime} \overline{\boldsymbol{m}}
$$

In addition to previously defined terms, $z$ is the $N \times 1$ column vector of nominal security holdings, with elements $z_{i}$. The variance of $\mathrm{Y}$ is:

$$
V(Y)=\sum_{i=1}^{N} \sum_{j=1}^{N} z_{i} z_{j} \sigma_{i j}=z^{\prime} C z
$$

Minimizing $V(Y)$ subject to a predetermined level of $E(Y)$ yields the following set of first order conditions for points on the risk-return efficiency frontier:

$$
2 C z-\lambda \bar{m}=0,
$$

where $\lambda$ is the Lagrange multiplier for the constraint.

Solving this system of equations for $z$ leads to the following expression for the vector of optimal holdings of risky assets along the efficiency frontier:

$$
2 z=\lambda C^{-1} \bar{m}
$$

Although the level of vector $z$ depends on the unknown $\lambda$-- and, therefore, gene:rally on the investor's utility function and the required expected return -- equation (4) does fix the raitios of the various risky assets along the efficiency frontier in any optimal portfolio: the famous portfolio separation theorem discovered by Tobin (1958). These optimal ratios will be preference-free, depending only on the investor's estimates of expected excess returns, and, once again, tire elements, $c_{i j}{ }^{-1}$, of the inverse of the covariance matrix: 


$$
\frac{z_{i}}{z_{j}}=\frac{\sum_{k=1}^{N} \bar{m}_{k} c_{i k}^{-1}}{\sum_{k=1}^{N} \bar{m}_{k} c_{j k}^{-1}}
$$

By combining equations (4), (1) and (2), one derives the investor's risk-return frontier -which haprens to be linear in expected return and standard deviation:

$$
E(Y)-r_{f} W=\left(\overline{\boldsymbol{m}}^{\prime} \boldsymbol{C}^{-1} \overline{\boldsymbol{m}}\right)^{1 / 2} \sqrt{V(Y)}
$$

Equations (4) and (5) and (6) emphasize the importance of the elements of the inverse of the covariance matrix $C$. Typically in portfolio analysis, however, we do not characterize these elements directly, but only indirectly, as those elements that map the original covariance matrix, $C$, into the identity matrix. The purpose of this note is to derive a direct characterization of the elements of $C^{-1}$, one that relies on a few key concepts and that leads to straightforward explanations of the optimal ratios in (5) and the slope of the risk-return locus in (6).

\section{Derivation}

We will start by adapting a useful partitioning technique developed by Anderson and Danthine (1981) for their study of hedging in futures markets. Partition the set of $N$ first order conditions (3) between the first equation and a $N-1$ equation block as follows:

$$
\begin{aligned}
\sigma_{11} z_{1}+\sum_{j=2}^{N} \sigma_{1 j} z_{j} & =\frac{\lambda}{2} \bar{m}_{1} \\
c_{.1} z_{1}+C_{N-1} z_{N-1} & =\frac{\lambda}{2} \bar{m}_{N-1}
\end{aligned} .
$$

The scaler variances, covariances, and levels of asset holdings (the $z_{j} s$ ), and excess return on the first asset $\left(\bar{m}_{1}\right)$ in the first equation are defined above. The matrix $C_{N-1}$ in the bottom block is the $N-1$ square subınatrix of the variance-covariance matrix $C$ formed by eliminating its first row and column; 
the first term of the bottom block, $c_{. l}$, is a $N-1 \times 1$ column vector comprising all but the fi:st element $\left(\sigma_{11}\right)$ of the first column of the original $C$ matrix (i.e. the covariances of the first asset with each of the remaining $N-1$ other assets); finally, $z_{N-1}$ and $\bar{m}_{N-1}$ are the $N-1$ column vectors made up of all but the first elements of the original $z$ and $\boldsymbol{m}$ vectors, respectively. Using this terminology, one can see that the summation term in the first equation of (6) equals the vector product: $c_{.1}^{\prime} z_{N-1}$. l.et us now solve the bottom block of $N$ equations for the $N-I \times l$ column vector $z_{N-1}$. Assuming the various inverses exist:

$$
z_{N-1}=\frac{\lambda}{2} C_{N-1}^{-1} \bar{m}_{N-1}-C_{N-1}^{-1} c_{.1} z_{1}
$$

This expression makes the holdings in $z_{N-1}$ (i.e. the levels of the holdings for assiets 2 through $N$ ) a function of the excess expected returns on all these other assets $\left(\bar{m}_{N-1}\right)$ and $z_{l}$, the holdings for asset 1. Note particularly the set of coefficients multiplying the level of $z_{l}:-C_{N-1}^{-1} c_{.1}$; this is the $(N-1) \times 1$ vector of least squares regression coefficients, $\beta$, when the excess expected return for asset 1 is regressed on the excess expected returns for the other $N-1$ assets. $^{2}$ Thus, insofar as the return on a given asset $j$ has a high positive coefficient in the regression for $r_{l}$, the holding for asset $j$ will be

2 The regression coefficients $-C_{N-1}^{-1} c_{.1}$ are easily related to the data-based regression coeffi:ients found in econometric texts. Thus, assume that there are $\mathrm{T}$ observations on the excess returns for each of the $\mathrm{N}$ variables. Following the partition introduced above, denote the $T$ by 1 vector of observed excess returns on the first asset by $\mathbf{y}$; also, denote, the T by $\mathrm{N}-1$ array of the vectors of excess returns on the other variables by $\mathbf{X}$. The sample-based estimate of the true variance-covariance matrix, $C$, would then be $X^{\prime} X$; similarly, the estimate of $c_{.1}$, the covariances of the first asset with all the $\mathrm{N}-1$ others, would be $\mathbf{X}^{\prime} \boldsymbol{y}$. Thus, the estimated regression coefficient $\hat{\boldsymbol{\beta}}$ would be $\left(\mathbf{X}^{\prime} \mathbf{X}\right)^{-1} \mathbf{X}^{\prime} \boldsymbol{y}$-- the typical expression in econometric texts and the empirical analog to $-C_{N-1}^{-1} c_{.1}$. 
correspondingly low. For future reference, it might be noted that the square of the multiple regression coefficient for the above regression equals $c_{.1}^{\prime} C_{N-1}^{-1} c_{.1} / \sigma_{11} .^{3}$

Let us now substitute this expression for $z_{N-1}$ into the first equation in system (7). We get an equation for the holdings of asset 1 :

$$
z_{1}\left[\begin{array}{llll}
\sigma_{11} & c_{.1}^{\prime} & C_{N-1}^{-1} & c_{.1}
\end{array}\right]=\frac{\lambda}{2}\left[\begin{array}{llll}
\bar{m}_{1} & -c_{.1}^{\prime} & C_{N-1}^{-1} & \bar{m}_{N-1}
\end{array}\right]
$$

As shown above, by substituting the appropriate regression coefficients and multiple correlation coefficient for the vector and matrix products appearing in (8), this expression for $z_{1}$ can be simplified as follows:

$$
z_{1} \sigma_{11}\left[1-R_{1}^{2}\right]=\frac{\lambda}{2}\left[\bar{m}_{1}-\beta^{\prime} \bar{m}_{N-1}\right]
$$

Since the original partition (6) can be carried out with any of the $N$ assets as the first, the holdings for any asset, $\mathrm{s}_{\mathrm{i}}$, can be expressed in the form of equation (9) above.

Equation (9) also provides us with the means to answer the primary question of this paper: how to provide a meaningful and direct characterization of the elements of the inverse matrix, $C^{-1}$. Recall that in equations (4), we have an alternative equation for each $z_{i}$ in terms of the elements of $C^{-1}$ and the excess return vector, $\bar{m}$. Our approach will be to use the known elements in the equation (9) for $z_{1}$ to solve for the unknown elements of the covariance matrix appearing in (4). To determine the first row of the $C^{-1}$ matrix, solve (9) for $z_{1}$ and subtract the alternative

3 The previous footnote shows that, in terms of textbook econometric notation, this term equals: $\boldsymbol{y}^{\prime} \boldsymbol{X} \hat{\boldsymbol{\beta}} / \boldsymbol{y}^{\prime} \boldsymbol{y}$, 'which equals the square of the multiple correlation coefficient of variable 1 (y) regressed on the $N-1$ other variab.es. For a discussion of this and earlier econometric points, see e.g., Johnston (1972), ch. 5, or Harvey (1990), ch. 2. 
equation for $z_{1}$, the first row of the set of equations (4). This leads to:

$$
0=\left[\frac{1}{\sigma_{11}\left(1-R_{1}^{2}\right)}-c_{11}^{-1}\right] \frac{\lambda m_{1}}{2}+\sum_{j=2}^{N}\left[-\frac{\beta_{j}}{\sigma_{11}\left(1-R_{1}^{2}\right)}-c_{1 j}^{-1}\right] \frac{\lambda m_{j}}{2}
$$

Since the excess expected returns, $\bar{m}_{j}$, may assume any value, the only way for (11) to hold is for each term in brackets to be identically equal to zero. Thus, $c_{11}^{-1}$ must equal the reciprocal of $\sigma_{11}\left(1-R_{1}^{2}\right)$-- that part of the variance of the first asset that cannot be diversified away by investing in the $N-I$ other assets (the asset's non-systematic risk). Similarly, the other elements of the first row, $c_{1 j}^{-1}$, must equal $-\beta_{1 j} / \sigma_{11}\left(1-R_{1}^{2}\right)-$ minus the regression coefficient for the $j$ th asset in the regression explaining the return on the first asset divided by that asset's non-systematic risk.

Since $C^{-1}$ is symmetric, the argument above is sufficient to determine the elements in both the first row and column. Although a certain measure of care must be taken in the proofs, it turns out that the same argument can be used to derive a version of equation (11) for asset levels $z_{2}$ through $z_{n}{ }^{4}$ The end result is that the diagonal elements of $C^{-1}$ equal the reciprocals of $\sigma_{i i}\left(1-R_{i}^{2}\right)$, where $R_{i}^{2}$ equals the multiple correlation coefficient for the regression of the return of the $i$ th stock on the set of all other returns. The off-diagonal elements, $c_{i j}^{-1}$, it turns out, can be expressed alternatively as the regression coefficient $-\beta_{i j} /\left[\sigma_{i i}\left(1-R_{i}^{2}\right)\right]$ or $-\beta_{j i} /\left[\sigma_{i j}\left(1-R_{j}^{2}\right)\right]$, the two expressions being equal.

The upshot of all of the above is the following direct characterization of the inverse of the

4 Clearly when we partition the first order conditions (7) differently, with another variable sh fted into the first column and row, the original $\mathbf{C}$ matrix is changed. Putting the first order condition for $z_{i}$ as the first row and column involves an even number of permutations of rows and columns of the original matrix; thus the determinants of the new and old system are equal. Similar statements hold for the other determinants and matrices leading to the expressions (8) and (10) for $z_{i}$. 
covariance matrix of returns for the set of $\mathrm{N}$ assets in question: ${ }^{5}$

$$
\left[\begin{array}{cccc}
\frac{1}{\sigma_{11}\left(1-R_{1}^{2}\right)} & -\frac{\beta_{12}}{\sigma_{11}\left(1-R_{1}^{2}\right)} & \cdots & -\frac{\beta_{1 N}}{\sigma_{11}\left(1-R_{1}^{2}\right)} \\
-\frac{\beta_{21}}{\sigma_{22}\left(1-R_{2}^{2}\right)} & \frac{1}{\sigma_{22}\left(1-R_{2}^{2}\right)} & \cdots & -\frac{\beta_{2 N}}{\sigma_{22}\left(1-R_{2}^{2}\right)} \\
\cdot & \cdot & \cdot & \cdot \\
\cdot & \cdot & \cdot & \cdot \\
-\frac{\beta_{N 1}}{\sigma_{N N}\left(1-R_{N}^{2}\right)} & -\frac{\beta_{N 2}}{\sigma_{N N}\left(1-R_{N}^{2}\right)} & \cdots & \frac{1}{\sigma_{N N}\left(1-R_{N}^{2}\right)}
\end{array}\right]
$$

\section{Implications}

\section{A. Asset Holdings}

Equation (12) shows that the many elements of the inverse matrix appearing in the expressions for the level (4) and ratio (5) of the dollar holdings of any asset $i$ can be succinctly related to three primary factors (in addition to the Lagrange multiplier): (1) positively to the asset's expected excess return over the riskfree rate, $\bar{m}_{i} ;(2)$ inversely to the non-diversifiable part of the asset's variance $\left[\sigma_{i i}\left(1-R_{i}^{2}\right)\right]-$ where $\left(1-R_{i}^{2}\right)$ is the percentage of the variance that cannot be explained by a linear combination of the returns on all other available assets; (3) positively or negatively to the expected

\footnotetext{
${ }^{5}$ That this matrix indeed is the inverse for the original covariance matrix $\mathbf{C}$ is verified most easily by relating the tlements of (12) to the determinants defining the traditional definition of $\mathbf{C}^{-1}$. An element, $\mathbf{c}^{-1}{ }_{\mathrm{w}}$, can be show: to be equal to the ratio of the cofactor $j i$ of the original covariance matrix $\left(\operatorname{Cof}_{i j}\right)$ and the determinant of $\mathbf{C}(\operatorname{det} C)$. (Since $\mathbf{C}$ is symmetric, the cofactor $j i$ equals the cofactor $i j$.) By adapting some results of Johnston (1972), p. $132 \mathrm{ff}$., one can relate the elements in matrix (12) to the above cofactors and determinant. Johnston, as an alternative to the normal econometric notation, relates normal regression coefficients and multiple correlation coefficients to the cofactors of the associated correlation matrix. One can use these results to show directly that $\operatorname{Cof}_{i i} / \operatorname{det} C$, the expression for $\mathrm{c}^{-1}$, equals $1 /\left[\sigma_{i k}\left(1-R_{i}^{2}\right)\right]$; similarly, $\mathrm{c}^{-1}{ }_{u}$, defined as $\operatorname{Cof} f_{i j} / \operatorname{det} C$, equals also $\left(\operatorname{Cof}_{i j} / \operatorname{Cof}_{i i}\right)\left(\operatorname{Cof}_{i i} / \operatorname{det} C\right)$, which equals $-\beta_{i j} /\left[\sigma_{i j}\left(1-R_{i}^{2}\right)\right]$.
} 
excess returns on other assets, $\bar{m}_{j}$, depending on the sign of the regression coefficient, $-\beta_{i j}$.

B. The Investor's Risk-Return Frontier

Equation (6), above, shows the investor's optimal tradeoff between risk and return to be linear with slope $\left(\bar{m}^{\prime} C^{-1} \bar{m}\right)^{1 / 2}$. The direct characterization of $C^{-1}$ in (12) shows us how to interpret this expression.

In the simplest case, a world with a single risky asset, $C^{-1}$ collapses to $1 / \sigma_{11}$ and the slope,

$\mathrm{dE} / \mathrm{dS}$ reduces to $m_{1} / \sqrt{\sigma_{11}}$, the only possible tradeoff between risk and return in such a world. The second simplest case involves adding a second risky asset, but with returns independent from the first.

This changes $\left(\bar{m}^{\prime} C^{-1} \bar{m}\right)^{1 / 2}$ and $\mathrm{dE} / \mathrm{dS}$ to the square root of $\frac{m_{1}^{2}}{\sigma_{11}}+\frac{m_{2}^{2}}{\sigma_{22}}$. If the investor optimizes --

in this case by taking a diversified portfolio -- the optimal tradeoff becomes a function of the expected excess returns of the two assets: the square of $\mathrm{dE} / \mathrm{dS}$ being a weighted average of the squared excess returns, the weights being the reciprocals of the variances of the respective assets. Because of the power of diversification, the investor's tradeoff between risk and return can easily be shiown to have improved (the slope increased). Because of the diagonality of the inverse matrix (12) in the case of independent asset returns, the above results are easily generalized to any number of assets. Thus, for $N$ assets:

$$
\bar{m}^{\prime} C^{-1} \bar{m}=\sum_{i=1}^{N}\left[m_{i}^{2} / \sigma_{i i}\right]
$$

The most realistic cases of course are those where the asset returns are correlated -- where the off-diagonal elements and the multiple correlation coefficients in (12) are non-zero. How the knowledge of $C^{-1}$ helps in the analysis can be illustrated by a consideration of the general 2-asset 
case. After some algebra, the general form of the risk-return tradeoff can be related to that for the independen:e case as follows:

$$
\bar{m}^{\prime} C^{-1} \bar{m}=\frac{m_{1}^{2}}{\sigma_{11}}+\frac{m_{2}^{2}}{\sigma_{22}}+\left[\frac{m_{1}^{2}}{\sigma_{11}\left(1-R_{1}^{2}\right)}+\frac{m_{2}^{2}}{\sigma_{22}\left(1-R_{2}^{2}\right)}-\frac{2 \beta_{12} m_{1} m_{2}}{\sigma_{11}\left(1-R_{1}^{2}\right)}\right]
$$

Whether the non-zero correlation between the two risky returns improves or worsens the investor's risk-return tradeoff, depends, relative to the independence case, on the sign of the sum inside the brackets. One immediate implication is that when the two assets are negatively correlated -- $\beta_{12}$ negative -- the tradeoff must improve. ${ }^{6}$

For a positive $\beta_{12}$, the analysis of (14) leads to the answer "it depends." For example, consider the case where the expected excess returns and variances of both assets are equal; since it is easily shown for this case that $R_{1}^{2}=R_{2}^{2}=\beta_{12}^{2}=\beta_{21}^{2}$, the numerator of the expression in brackets simplifies to $2 m^{2} \beta_{12}\left(\beta_{12}-1\right)$. In this special case, however, $\beta_{12}$ must be less than one, so any positive covariance will worsen the risk-return tradeoff.

That this last result is not general can be seen by considering specific values when the excess returns of the two asset are allowed to diverge. A typical case leading to a positive sign on the bracketed terms in (14) is: $\beta_{12}=.95, m_{1}=10, m_{2}=2$, with $\sigma_{11}=\sigma_{22}$. The explanation for this improvement in the risk-return tradeoff over the independence case can be understood by using equation (4) and the elements of $C^{-1}$ to look at the optimal holdings of the two assets for the above values. It turns out that the optimal portfolio in this case specifies a short position in asset 2 . With the high positive covariance between the two returns, the optimizing investor can control the risk

6 This statement assumes that the excess expected returns of both assets are positive. In the 2 variable case, of course, the: covariance $\sigma_{12}$ determines the sign of both regression coefficients, $\beta_{i j}=\sigma_{i j} / \sigma_{i j}$. 
buildup of a heavy investment in the high-return asset by going short in the other.

\section{Linkages to the Capital Asset Pricing Model}

The assumptions of the CAPM do not admit short positions, so the slope of the risk-return locus, $\bar{m}^{\prime} C^{-1} \bar{m}$, that led to the previous result would be inadmissible in this context; typically the excess returns would adjust until all assets would be held in long positions. It is not haril to show how the equilibrium risk-return locus determined by the CAPM is related to $\bar{m}^{\prime} C^{-1} \bar{m}$. A major

result of the CAPM is that the market portfolio, with expected return (per dollar) $r_{M}$ and standard deviation $\sigma_{M}$, lies on the risk-return efficiency frontier; given that the slope of the frontier is a constant, it must, therefore, be equal to $\left(r_{M}-r_{f}\right) / \sigma_{M}$. Under the assumptions of the CAPM -- among them the common beliefs as to expected returns, variances, and covariances -- each investor must share the same risk-return frontier. Thus:

$$
\left(r_{M}-r_{f}\right) / \sigma_{M}=\left(\bar{m}^{\prime} C^{-1} \bar{m}\right)^{1 / 2}
$$

Although the knowledge of the inverse matrix is related to and provides a useful characterization of the slope of the CAPM market line, it should be noted that, unlike the case in the previous section, the analysis of the effects of changes in the elements of $C^{-1}$ on the risk-return frontier is no longer straightforward. This is because, for the general equilibrium relationship expressed by the CAPM line, the excess expected returns making up the $\overline{\boldsymbol{m}}$ vector are now endogenous variables; thus, any exogenous change in an element of the inverse matrix will also result in changes in some or all of the excess expected returns.

Other key concepts and relationships in the CAPM can be related to the elements of $C^{-1}$. Using equation (15), the equilibrium expression for the excess return on any security -- the CAPM 
security murket line -- can be restated as follows: ${ }^{7}$

$$
\bar{r}_{1}-r_{f}=\left[\frac{\left(\bar{r}_{M}-r_{f}\right)}{\sigma_{M}}\right] \frac{\sigma_{i M}}{\sigma_{M}}=\left[\left(\overline{\boldsymbol{m}}^{\prime} C^{-1} \overline{\boldsymbol{m}}\right)^{1 / 2}\right] \frac{\sigma_{i M}}{\sigma_{M}},
$$

where $\sigma_{i M}$ is the covariance of security $i$ with the market portfolio.

The expression $\sigma_{i M} / \sigma_{M}$ can be shown to be the effect on the risk of the market portfolio of a marginal dollar in asset $i$, while $\sigma_{i M} / \sigma_{M}^{2}$ is the security's beta, $\beta_{i}$. The key to reinterpreting these concepts in terms of the elements of $C^{-1}$ is that the optimal security holdings of any investor can be expressed as a multiple of the market portfolio; thus, the vector of holdings equal to the market portfolio satisfies the optimal equations (3), (4), (5) and especially (7). The first row of the partition (7) is particularly useful, because when one substitutes the market portfolio for the $z_{i} \mathrm{~s}$ in that row, the left hand side becomes $\sigma_{1 M}$-- expressible as the product of the row vector of all the covariances of the first security, say $\sigma_{1}$, and the market portfolio. ${ }^{8}$ Further, since the market portfolio satisfies the marginal ccnditions (4) for some Lagrange multiplier, $\lambda * \sigma_{1 M}$ equals $\left(\lambda^{*} \sigma_{1} C^{-1} \bar{m} / 2\right)$; moreover, using (4) arid substituting into the equation for the variance (2), the standard deviation of the market portfolio, $\sigma_{M}$, equals the same Lagrange multiplier times $\left(\bar{m}^{\prime} C^{-1} \bar{m}\right)^{1 / 2}$. We thus have the alternative

7 See, e g., Copeland and Weston (1983), pp. 187-189 or Elton and Gruber (1987), chapter 11, for good expositions and derivations of the security market line and its components.

${ }^{8}$ It might be worth noting that this first equation, which shows that the excess return on the first asset is proportional to $\sigma_{1 M}$, can be used as a starting point for the derivation of the security market line. Moreover, since any asset can be chosen as the first, the above relationship holds generally. 
expression, in terms of the elements of $C^{-1}$, for the marginal risk of security $i$ :

$$
\frac{\sigma_{i M}}{\sigma_{M}}=\frac{\sigma_{i} C^{-1} \bar{m}}{\left(\bar{m}^{\prime} C^{-1} \bar{m}\right)^{1 / 2}} .
$$

Similarly, $\boldsymbol{\beta}_{\boldsymbol{i}}$ equals:

$$
\beta_{i}=\frac{\sigma_{i M}}{\sigma_{M}^{2}}=\frac{2 \sigma_{i .} C^{-1} \bar{m}}{\lambda \cdot \bar{m}^{\prime} C^{-1} \bar{m}} .
$$




\section{References}

Anderson, Ronald W. and Jean-Pierre Danthine, "Cross Hedging," Journal of Political Economy, December 1981, 89, 1182-1196.

Copeland, 'Thomas E. and J. Fred Weston, Financial Theory and Corporate Policy, 2nd. ed., Reading Mass.: Adclison Wesley, 1983.

Elton, Edwin J. and Martin J. Gruber, Modern Portfolio Theory and Investment Analysis, 3rd ed., New York: Wiley, 1987.

Harvey, Arddrew C., The Econometric Analysis of Time Series, 2nd ed., Cambridge: MIT, 1990.

Johnston, J , Econometric Methods, 2nd. ed., New York: McGraw-Hill, 1972.

Mossin, Jan, Theory of Financial Markets, Englewood Cliffs, N.J.: Prentice-Hall, 1973.

Tobin, James, "Liquidity Preference as Behavior Towards Risk," The Review of Economic Studies, February 1958, 25, 65-86. 


\section{International Finance Discussion Papers}

IFDP

Number
Titles

1995

528 On the Inverse of the Covariance Matrix in Portfolio Analysis

527 International Comparisons of the Levels of Unit Labor Costs in Manufacturing

526 Uncertainty, Instrument Choice, and the Uniqueness of Nash Equilibrium: Microeconomic and Macroeconomic Examples

525 Targeting Inflation in the 1990s: Recent Challenges

524 Economic Development and Intergenerational Economic Mobility

523 Human Capital Accumulation, Fertility and Growth: A Re-Analysis

522 Excess Returns and Risk at the Long End of the Treasury Market: An EGARCH-M Approach

521 The Money Transmission Mechanism in Mexico

$520 \quad$ When is Monetary Policy Effective?

519 Central Bank Independence, Inflation and Growth in Transition Economies

518 Alternative Approaches to Real Exchange Rates and Real Interest Rates: Three Up and Three Down

517 Product market competition and the impact of price uncertainty on investment: some evidence from U.S. manufacturing industries

Peter Hoopier

Elizabeth V/rankovich

Dale W. Henderson

Ning S. Ztıu

Richard T. Freeman Jonathan L. Willis

Murat F. Iyigun

Murat F. Iyigun

Allan D. Brunner
David P. Simon
Martina Copelman
Alejandro M. Werner
John Ammer
Allan D. Brunner
Prakash Loungani
Nathan Shtets
Hali J. Edison
William R. Melick
Vivek Ghosal
Prakash Loungani
Jon Faust
Ralph Tryon

Guy V.G. Stevens

Allan D. Brunner

Martina Copelman

John Ammer

Ralph Tryon

Please address requests for copies to International Finance Discussion Papers, Division of International Finance, Stop 24, Board of Governors of the Federal Reserve System, Washington, DC 20551. 


\section{International Finance Discussion Papers}

IFDP

Number

Titles

Author(s)

1995

515 Supply-side sources of inflation: evidence

Prakash Loungani

from OECD countries

Phillip Swagel

514 Capital Flight from the Countries in Transition:

Some Theory and Empirical Evidence

Nathan Sheets

513 Bank Lending and Economic Activity in Japan:

Did "Financial Factors" Contribute to the Recent

Downturn?

Allan D. Brunner

Steven B. Kamin

512 Evidence on Nominal Wage Rigidity From a Panel

of U.S. Manufacturing Industries

Vivek Ghosal

Prakash Loungani

511 Do Taxes Matter for Long-Run Growth?: Harberger's Superneutrality Conjecture

Enrique G. Mendoza

Gian Maria Milesi-Ferretti

Patrick Asea

510 Options, Sunspots, and the Creation of Uncertainty

509 Hysteresis in a Simple Model of Currency Substitution

David Bowman

Jon Faust

Martin Uribe

508 Import Prices and the Competing Goods Effect

Phillip Swagel

507 Supply-side Economics in a Global Economy

Enrique G. Mendoza

Linda L. Tesar

506 The Lucas Critique In Practice: Theory Without Measurement

Neil R. Ericsson

John S. Irons

505 Real Exchange Rate Targeting and Macroeconomic Instability

Martin Uribe

504 Inferences from Parametric and Non-Parametric Covariance Matrix Estimation Procedures

Wouter J. Den Haan

Andrew T. Levin

Martin Uribe

Initial Real Effects of Credible Plans

John Ammer

Diversification: An Application to the Equity

Markets of Europe, Japan, and North America

Jianping Mei 